\title{
Panorama dos Inquéritos Ocupacionais no Brasil (2005-2015):uma revisão sistemática da literatura
}

\author{
Overview of Occupational Surveys in Brazil (2005-2015): \\ a systematic review of the literature
}

Tatiana Gobbi Mendes de Castro (https://orcid.org/0000-0003-4539-0287) ${ }^{1}$

Eduardo de Paula Lima (https://orcid.org/0000-0001-8957-8025) ${ }^{1}$

Ada Ávila Assunção (https://orcid.org/0000-0003-2123-0422) ${ }^{1}$

${ }^{1}$ Departamento de Medicina Preventiva e Social, Faculdade de Medicina, Universidade Federal de Minas Gerais. Av. Alfredo Balena 190/630, Santa Efigênia. 30310-150 Belo Horizonte MG Brasil. tatigob@hotmail.com

\begin{abstract}
Occupational Surveys are methods of collecting data from the cross-sectional studies, which are indispensable for the elaboration of public policies oriented to workers' health. The objective of this study was to perform a systematic review on articles that featured results of Occupational Surveys conducted in Brazil from 2005 to 2015. Based on the Prisma recommendation, this review selected articles indexed in Medline, Lilacs and Scielo databases. Analysis of the data consisted of the description of the Occupational Surveys according to: large regions in which the research was carried out; techniques and instruments used; topics covered; sectors of the economy in which health-work relations were investigated; and methodological criteria. Differences in the distribution of Occupational Surveys were observed regionally and by sector. The structure of the investigations, for the most part, was concerned with identifying sickness in workers. A lack of consensus regarding the methods used was revealed, which consequently prejudiced the comparison of results and monitoring of health and working conditions. Efforts are needed to build consensus and foster research in sectors and regions that are as yet scantly explored.

Key words Epidemiological surveys, Worker's health, Cross-sectional studies, Working conditions
\end{abstract}

Resumo Inquéritos Ocupacionais são métodos de coleta de dados dos estudos transversais indispensáveis à elaboração de políticas públicas voltadas à saúde do trabalhador. O objetivo deste estudo foi realizar uma revisão sistemática sobre artigos que retratavam resultados de Inquéritos Ocupacionais conduzidos no Brasil, no período de 2005 a 2015. Baseada na recomendação Prisma, esta revisão selecionou artigos indexados em base de dados Medline, Lilacs e SciELO. A análise dos dados consistiu na descrição dos Inquéritos Ocupacionais de acordo com: grandes regiões nas quais as pesquisas foram realizadas, técnicas e instrumentos utilizados, temas abordados, setores da economia nos quais as relações saúde-trabalho foram investigadas e critérios metodológicos. Observou-se diferenças na distribuição dos Inquéritos Ocupacionais regional e setorialmente. A estrutura das investigações, em sua maioria, se interessava em identificar adoecimento em trabalhadores. Ausência de consenso no tocante aos métodos utilizados foi observada com prejuízos para a comparação de resultados e monitoramento da saúde e condições de trabalho. Esforços são necessários para a elaboração de consensos e fomento à pesquisa em setores e regiões ainda pouco exploradas.

Palavras-chave Inquéritos epidemiológicos, Saúde do trabalhador, Estudos transversais, Condições de trabalho 


\section{Introdução}

A forma em que os grupos se inserem na força de trabalho influencia as chances de acesso aos recursos econômicos e sociais, e afeta a situação de saúde dos seus membros. As condições sob a quais os indivíduos operam durante suas jornadas geram efeitos físicos e psíquicos que estão relacionados ao adoecimento, afastamento do trabalho e morte ${ }^{1}$.

Inquéritos Ocupacionais são investigações em saúde que utilizam métodos estatísticos para a análise dos dados coletados em populações de trabalhadores. São estudos transversais que fornecem informações sobre saúde e trabalho, de maneira a viabilizar a elaboração de estratégias de prevenção e promoção da saúde de um grupo ocupacional e formulação de políticas setoriais ${ }^{2}$. Trata-se de um desenho epidemiológico valioso para se conhecer as características dos trabalhadores ${ }^{3}$. Fornecem informações que não são passíveis de acesso por outras vias ${ }^{4}$. O objetivo desses inquéritos é obter uma "fotografia" da situação de saúde e dos fatores de risco aos quais a população trabalhadora está exposta. Em sua maioria, são realizados por meio de questionários e entrevistas. A abrangência pode ter alcance internacional, nacional, regional ou local. Espera-se que a amostra seja representativa da população alvo.

Experiências anteriores nos países da União Europeia e na América Central trouxeram aportes relevantes tanto para ampliar o conhecimento sobre a situação de saúde dos trabalhadores quanto para elaboração de hipóteses de pesquisa mais adequadas ao cenário de transformação do modo de produção em escala mundial ${ }^{5,6}$.

Dados nacionais colhidos nos domicílios identificam mais de $50 \%$ da força de trabalho ocupada sem contratos formais, sendo que essa proporção é maior nas regiões Norte e Nordeste $(\text { IBGE })^{7}$. A geração de empregos no País continua ocorrendo abaixo do seu potencial. A polarização entre um segmento formal e outro informal é crescente. Nas últimas décadas, houve avanço nos processos de privatizações, terceirização das atividades, informalização e precarização das ocupações ${ }^{8}$. O arcabouço jurídico-legal foi recentemente modificado, de maneira a diminuir os custos trabalhistas não salariais, permitir adaptações das normas contratuais, e interferir na estrutura sindical.

Resultados de pesquisas realizadas em outros países evidenciam insegurança para o empregado que está contratado temporariamente e com menos acesso aos direitos. São situações que aumen- tam a chance de insatisfação, sintomas psíquicos e outras morbidades 9 . Os empregados na informalidade em sua maioria são jovens, mulheres, não brancos, e que têm rendimentos mais baixos e menos benefícios comparados aos seus antônimos. Independentemente do setor, os trabalhadores terceirizados ou sob contratos temporários frequentemente são menos livres para escolher o período de suas férias e, raramente, são representados nos comitês de saúde. Além disso, eles ocupam funções com menor exigência de habilidade, mais risco e experimentam pior resultado de saúde, quando comparados aos trabalhadores permanentes. Sabe-se, também, que dispõem de menos informação sobre o seu ambiente, usufruem de menos autonomia e controle sobre os horários, quando comparados aos colegas empregados sob vínculo permanente ${ }^{10,11}$. No conjunto, as situações citadas expressam a miríade de vias pelas quais as diferenças sociais se associam com cada dimensão das desigualdades em saúde relacionadas à ocupação e às condições de trabalho ${ }^{12}$. Abre-se assim o debate sobre os limites das medidas tradicionais da posição socioeconômica, tendo em vista as constatações sobre modelo de desenvolvimento econômico que incide sobre a estrutura e a inserção da força de trabalho no mercado ${ }^{1,10}$.

Trabalhadores empregados provavelmente encontram-se expostos a riscos emergentes derivados da intensificação das exigências físicas e psicossociais das tarefas geradas pelas novas conformações das relações de trabalho ${ }^{13}$. Prevalências de morbidades acima ou abaixo do encontrado na população em geral, como foi o caso da depressão na amostra dos bombeiros de Belo Horizonte ${ }^{14}$, convocam novos dispositivos de interpretação.

Recentemente, grupos transnacionais investem em estratégias para facilitar a comparação dos resultados obtidos ${ }^{3,15,16}$, haja vista a necessidade de gerar consensos e padronizações nos procedimentos de coleta de dados. Os autores propugnam os critérios técnicos sobre os instrumentos de coleta e suas utilizações (questionário, desenho amostral e a forma de coletar informações) para melhorar a qualidade e validade dos Inquéritos Ocupacionais ${ }^{17,18}$. Se, por um lado, o conceito de processo de trabalho ou a noção de condições de trabalho têm se mostrado adequados para embasar as hipóteses de nossos empreendimentos investigativos, por outro lado, diante das explanações sobre novo perfil de adoecimento e das transformações macroestruturais citadas, convém ajustar o horizonte teórico-conceitual dos modelos explicativos. 
Há quase duas décadas, Gomez e Lacaz $^{19}$ alertaram sobre a fragmentação e dispersão da produção científica da área com consequentes déficits de subsídios para apoiar as formulações que buscam melhorar a saúde dos trabalhadores brasileiros. Como se desenvolveram desde então as pesquisas na área de saúde do trabalhadores? O objetivo deste estudo foi realizar uma revisão sistemática da estrutura e a da qualidade técnica dos artigos que focaram Inquéritos Ocupacionais conduzidos no Brasil, cujos resultados foram publicados em veículos disponíveis em bases indexadas.

\section{Metodologia}

A estrutura dessa pesquisa tomou como referência o Preferred reporting items for systematic reviews and meta-analyses (Prisma) que consiste em um checklist de 27 itens e um fluxograma de quatro etapas. Ainda que a maior força do Prisma seja a otimização das revisões sistemáticas e meta-análises com ênfase em resultados empíricos, é também uma referência para outros tipos de estudos, os quais processam adaptações pertinentes ${ }^{20}$.

A composição do corpus foi feita através da seleção de artigos que relataram resultados de Inquéritos Ocupacionais. A busca foi limitada às pesquisas sobre saúde e trabalho. Inicialmente, foram localizadas publicações nas bases Medical Literature Analysis and Retrieval System Online (Medline) (via PubMed), Literatura Latino-Americana e do Caribe em Ciências da Saúde (Lilacs) (via BVS) e Scientific Electronic Library Online (Scielo). As estratégias de busca foram adaptadas para cada base, de acordo com as ferramentas disponíveis, cujos descritores nos idiomas português, inglês e espanhol foram selecionados em listas disponíveis nas bases de dados e nos artigos examinados na fase preliminar. Para contornar o campo de interesse, utilizou-se a combinação dos descritores "condições de trabalho" e "saúde do trabalhador”. Na sequência, foram identificados descritores mais específicos, como "acidentes de trabalho", "doenças profissionais", "questionários", "estudos transversais" e "inquéritos de saúde", os quais permitiram abarcar o maior número possível de inquéritos ocupacionais. De posse do conjunto, decidiu-se por construir grupos de descritores próprios ao foco da pesquisa: o primeiro relacionado à saúde do trabalhador ("saúde do trabalhador", "condições de trabalho", "acidentes de trabalho" e "doenças profis- sionais") e o segundo condizente ao método de estudo utilizado pelos autores ("inquéritos de saúde", "questionários” e "estudos transversais"). Para a combinação desses grupos de descritores foram utilizados os operadores booleanos "OR" e "AND", em respeito às especificidades de cada base examinada.

Após leitura dos títulos e resumos dos artigos, foram incluídos no corpus da pesquisa artigos científicos disponíveis e publicados nos idiomas citados, entre os anos de 2005 e 2015, com desenhos transversais e do tipo quantitativo. Buscouse localizar estritamente artigos realizados em grupos de trabalhadores atuando no Brasil independente da nacionalidade dos sujeitos amostrados. Foram excluídas as publicações nas quais não constavam resumo e aquelas que, apesar de abordar grupos ocupacionais, não enfocaram desfechos relacionados à saúde, por exemplo, abordagens sobre gestão ou desempenho. Privilegiando o autorrelato, foram excluídos artigos que empregaram métodos de aferição direta (pressão arterial, peso e altura, por exemplo) ou coleta de material para exames laboratoriais.

Para a elegibilidade procedeu-se a leitura completa das publicações previamente selecionadas, com enfoque na desenho do estudo (inquérito). Nessa etapa, as características de cada inquérito foram devidamente registradas em um caderno de notas. Por meio de idas e vindas, o caderno de notas viabilizou a definição do corpus, a extração e a classificação dos dados em três categorias: características gerais, composição dos inquéritos e critérios de qualidade. As primeiras dizem respeito ao tipo de publicação, local onde o estudo foi realizado (por grande região do Brasil), população de interesse de acordo com os grupos de atividade econômica seguindo a classificação da Pesquisa Nacional de Amostra de Domicílios Contínua (PNADC) e temas estudados nos inquéritos. Na categoria composição foram examinados os instrumentos e a modalidade da coleta (face a face, telefone, correios, internet, etc.). Validação do instrumento utilizado, realização de estudo piloto e descrição do cálculo amostral foram os critérios para avaliar a qualidade de cada publicação do corpus.

\section{Resultados}

A Figura 1 ilustra o processo de seleção das publicações do corpus, à luz do modelo Prisma.

De acordo com a estratégia adotada, foram identificados 1.231 artigos em bases de dados 


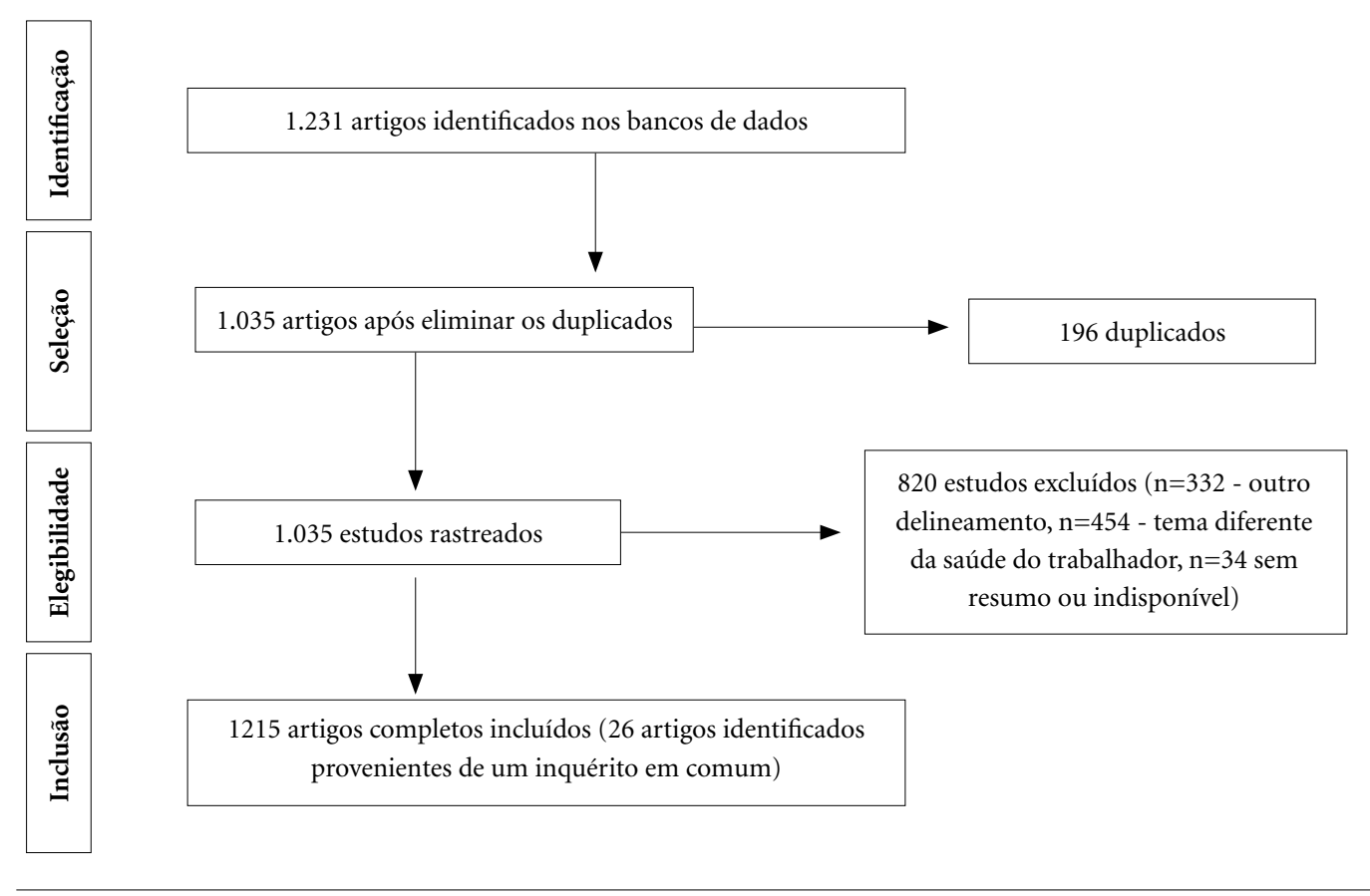

Figura 1. Fluxograma do processo de seleção de estudos realizados por meio de inquéritos.

Fonte: Moher et al. ${ }^{20}$, adaptado pela autora.

disponíveis na internet. Na seleção de inquéritos, foram excluídos os artigos duplicados $(n=196)$, os que não tinham utilizado desenho transversal e abordagem quantitativa $(n=140)$, aqueles com temas díspares ao interesse da pesquisa $(n=365)$ e artigos que não incluíram o resumo no texto $(\mathrm{n}=$ 17). Ao final, 513 publicações foram selecionadas.

$\mathrm{Na}$ etapa seguinte, procedeu-se a leitura completa dos textos, sendo excluídos outros 298 artigos pelos mesmos critérios utilizados na etapa anterior (192 não eram estudos transversais, 89 versavam sobre temas alheios à saúde dos trabalhadores e 17 não exibiam resumo ou não estavam disponíveis na íntegra. Identificaramse inquéritos que foram objeto de mais de uma publicação $(n=26)$. Encerrou-se a etapa com 215 artigos selecionados, entre as quais 122 foram extraídas da base de dados Medline (via Pubmed), 77 da base Lilacs (via BVS) e 16 da base Scielo.

O Gráfico 1 ilustra o crescimento no número de artigos entre 2005 e 2015. Em 2006 observa-se um salto em relação a 2005 (de 2 para 15). Entre 2006 e 2015 o crescimento é contínuo, embora não linear (66,7\%). O ano de 2012 apresenta o maior volume $(n=33)$.
Quanto à distribuição geográfica, desponta a região Sudeste onde foi realizada aproximadamente a metade $(44,2 \%)$ dos Inquéritos Ocupacionais no período analisado, seguida da região Sul (20,9\%) e Nordeste (17,7\%) (Figura 2).

Em relação ao ramo de produção, a maioria $(66,0 \%)$ diz respeito à "Administração pública, defesa, seguridade social, educação, saúde humana e serviços sociais", com destaque para a saúde humana $(n=94)$ e educação $(n=30)$ que, se reunidas, representam mais de $50 \%$ das publicações. Os grupamentos "Indústria geral de transformação" e "Transporte, armazenagem e correio" representam, respectivamente $9,4 \%$ e $10,2 \%$ das populações estudadas nos inquéritos. Apenas uma publicação abordou grupamentos no ramo de "Alojamento e alimentação" e "Comércio, reparação de veículos automotores e motocicletas". O grupamento "Outros serviços" englobou grupos que não se enquadravam nos demais, como: cabeleireiros, trabalhadores adolescentes, trabalhadores urbanos, tropeiros/coletores de materiais recicláveis, profissionais do sexo, trabalhadores do serviço de limpeza, e trabalhadores do setor de frigorífico (Tabela 1). 


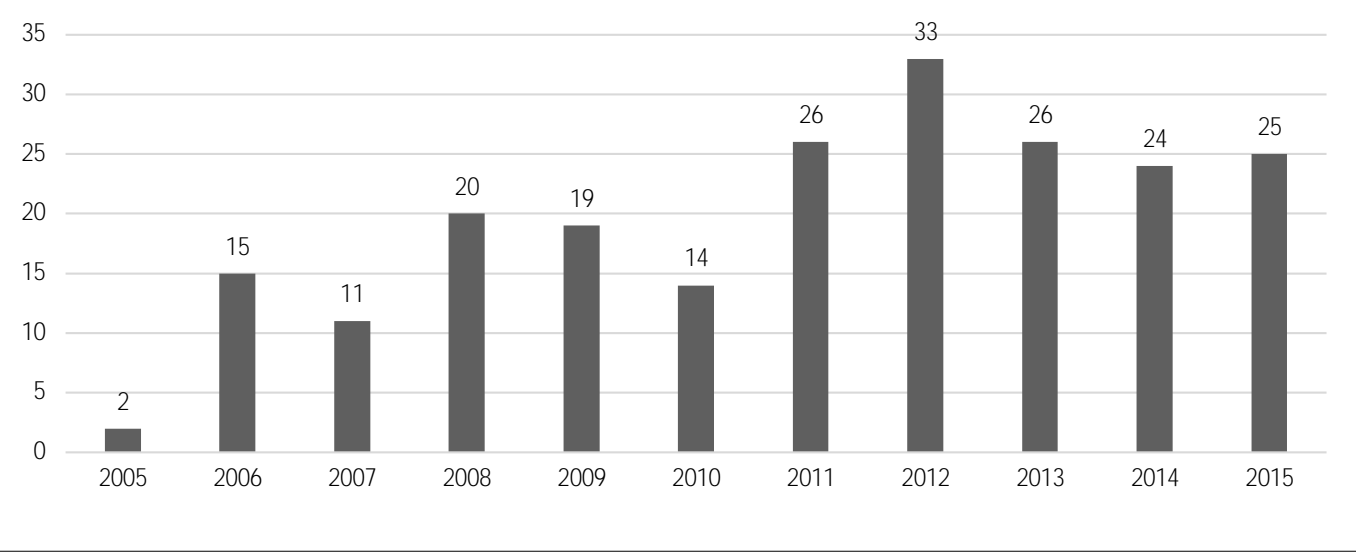

Gráfico 1. Distribuição dos artigos publicados sobre Inquéritos Ocupacionais de acordo com o ano de publicação (2005 a 2015).

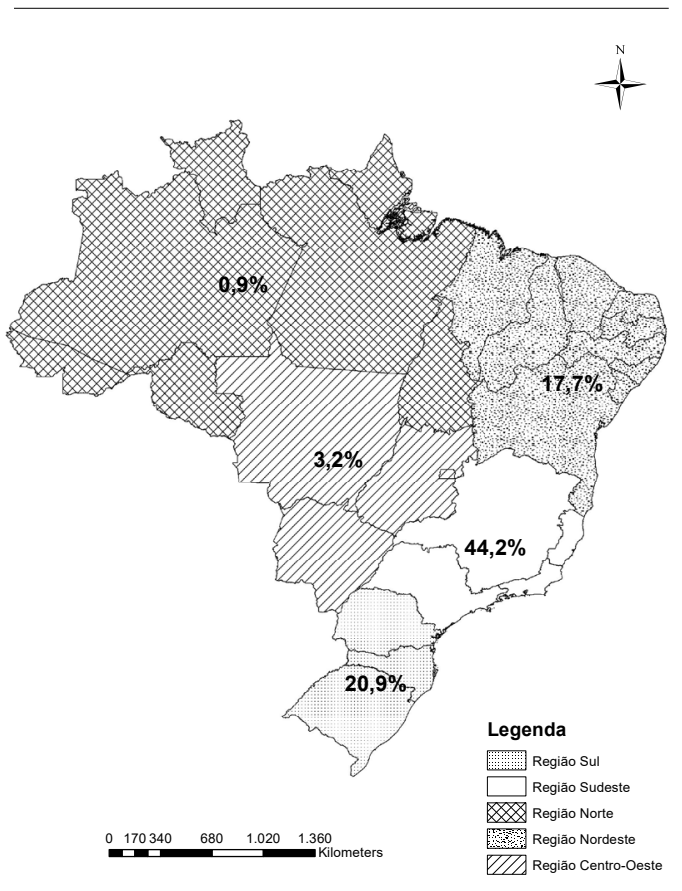

Figura 2. Distribuição dos artigos $(n=215)$ que publicaram resultados de Inquéritos Ocupacionais no Brasil (2005-2015) de acordo com a grande região em que a pesquisa foi conduzida.

Fonte: Dados da pesquisa

Quanto aos parâmetros de qualidade, 28,4\% das publicações citaram a realização de estudo piloto, $53,5 \%$ validaram o instrumento de coleta de dados, $37,2 \%$ descreveram os procedimen- tos para o cálculo amostral. Vale mencionar que $10,2 \%$ dos artigos relataram resultados de censos realizados em populações específicas.

Em relação à coleta de dados, $42,8 \%$ utilizaram as técnicas de entrevista face a face, $33,0 \%$ utilizaram a técnica do autopreenchimento. Uso da internet, correios ou ambos representaram 4,7\% das publicações analisadas.

Diferentes temas foram tratados pelos autores. A maioria objetivou investigar a ocorrência de morbidades, com predomínio de problemas mentais, doenças musculoesqueléticas e problemas vocais e auditivos. Nove artigos trataram de acidentes de trabalho. Sobre exposição ambiental (Condições de trabalho e Exposição ocupacional) identificaram-se 14 publicações. Vinte e oito artigos trataram de aspectos como Capacidade para o trabalho, Educação, promoção e proteção, Hábitos e estilos de vida. Descrição de Situação de saúde e absenteísmo e violência foram abordados em 35, 5 e 3 artigos, respectivamente (Tabela 2).

\section{Discussão}

A formulação de políticas públicas de saúde para a população de trabalhadores depende da disponibilidade de informações válidas e consistentes. Os inquéritos são meios reconhecidos para fornecer subsídios aos agentes formuladores desse tipo de políticas, como vem sendo praticado no Brasil para monitorar doenças crônicas, por exemplo $^{21}$. Os resultados da análise de 215 arti- 
gos publicados entre 2005-2015 levam a constatar nítido crescimento de publicações a cada ano. A tendência é confirmada quando se analisa a produção, independente do tema, na grande área da saúde, que também se beneficiou do aumen-

Tabela 1. Distribuição dos Inquéritos Ocupacionais de acordo com a população de estudo, classificada por grupamento de atividade econômica.

\begin{tabular}{lcc}
\hline \multicolumn{1}{c}{$\begin{array}{c}\text { Grupamento de atividade } \\
\text { econômica }\end{array}$} & N & \% \\
\hline $\begin{array}{l}\text { Agricultura, pecuária, produção } \\
\text { florestal, pesca e aquicultura }\end{array}$ & 6 & 2,8 \\
$\begin{array}{l}\text { Indústria geral Indústria de } \\
\text { transformação }\end{array}$ & 20 & 9,3 \\
$\begin{array}{l}\text { Comércio, reparação de veículos } \\
\text { automotores e motocicletas }\end{array}$ & 1 & 0,5 \\
$\begin{array}{l}\text { Construção } \\
\text { Transporte, armazenagem e correio }\end{array}$ & 22 & 10,2 \\
$\begin{array}{l}\text { Alojamento e alimentação } \\
\text { Informação, comunicação e } \\
\text { atividades financeiras, imobiliárias, } \\
\text { profissionais e administrativas }\end{array}$ & 1 & 0,5 \\
$\begin{array}{l}\text { Administração pública, defesa, } \\
\text { seguridade social, educação, saúde } \\
\text { humana e serviços sociais }\end{array}$ & 142 & 66,0 \\
$\begin{array}{l}\text { Outros serviços } \\
\text { Várias grupos }\end{array}$ & & \\
Total & 10 & 4,7 \\
\hline
\end{tabular}

Tabela 2. Distribuição dos temas estudados nos Inquéritos Ocupacionais selecionados do corpus.

\begin{tabular}{lcc}
\multicolumn{1}{c}{ Temas } & N & \% \\
\hline Saúde mental e aspectos & 76 & 35,3 \\
psicossociais & & \\
Situação de saúde & 35 & 16,3 \\
Doenças musculoesqueléticas & 27 & 12,6 \\
Educação, proteção e promoção a & 15 & 7,0 \\
saúde & & \\
Problemas vocais e auditivos & 12 & 5,6 \\
Exposição ocupacional & 10 & 4,6 \\
Acidente & 9 & 4,2 \\
Capacidade para o trabalho & 8 & 3,7 \\
Condições de trabalho & 4 & 1,9 \\
Outros & 6 & 2,8 \\
Absenteísmo & 5 & 2,3 \\
Hábitos e estilos de vida & 5 & 2,3 \\
Violência & 3 & 1,4 \\
Total & 215 & 100 \\
\hline
\end{tabular}

to dos recursos públicos destinados à pesquisa e desenvolvimento, que passou de sete milhões, em 2005, para 25 milhões, em $2013^{22}$.

Observaram-se diferenças regionais, com nítida vantagem para a região Sudeste, de maneira coerente à distribuição das instituições de pesquisa no País ${ }^{23}$. Em 2014, o Brasil contava com 2.368 instituições de ensino superior, sendo 1.126 localizadas na região Sudeste e apenas 149 na região $\mathrm{Norte}^{24}$. O número de mestres e doutores formados e atuantes no País segue a mesma distribuição: 49\% do total de mestres e 60\% dos doutores titulados, em 2014, estavam inseridos em instituições localizadas na região Sudeste ${ }^{25}$. Disparidades regionais na concentração dos estudos ocupacionais provavelmente tornam menos visíveis os grupos mais vulneráveis - os desempregados, os subempregados, os trabalhadores rurais e em outras situações de precariedade - que geralmente vivem e trabalham nas regiões de menor ritmo acadêmico e científico.

Apenas um dos artigos examinados abordou dimensões do emprego (jornada de trabalho) como variável desfecho. Apesar das fortes evidências que explicam na atualidade as desigualdades na distribuição das doenças crônicas nos adultos relacionadas aos estilos menos saudáveis, que, por sua vez, são influenciados pelo tipo de inserção na força de trabalho ou pela intensificação do ritmo laboral ${ }^{1}$, foram raros os inquéritos que adotaram o tema como desfecho. Não foi identificado nenhum artigo de abrangência nacional.

Fragilidades técnicas como deficiências no planejamento amostral foram recorrentes nos artigos examinados. Observou-se a ausência de consenso no tocante aos procedimentos para realização de estudo piloto e fragilidades ou ausência de validação de instrumentos. Tem-se um alerta quanto ao "descuido" nesses quesitos fundamentais para garantir a reprodutibilidade $\mathrm{e}$ continuidade ${ }^{3,17,26,27}$.

Em relação à estratégia de coleta de dados, uma fração exígua utilizou as tecnologias disponíveis (telefone e Internet) que garantem mais agilidade e menos despesas na realização da coleta ${ }^{17}$, com crescente adesão dos pesquisadores da grande área da saúde. Tem-se assim mais um elemento para reflexão dos grupos com expertise em inquéritos ocupacionais.

A considerar a estrutura econômica em que os serviços empregam mais trabalhadores e são responsáveis por quase $70 \%$ do Produto Interno Bruto (PIB), não foi surpreendente identificar $70 \%$ dos artigos focados no referido setor com predominância de análises dos grupos inseridos 
nos estabelecimentos de saúde ou no setor educacional.

Mais da metade dos artigos descreveram prevalências de doenças e sintomas psíquicos, gerando uma sequência de confirmações de hipóteses já estabelecidas. De fato, os inquéritos em saúde têm o objetivo de descrever frequências e fatores associados a morbidades. Verificou-se escassez de estudos que se interessaram em conhecer a situação de saúde geral. A predominância de estudos cujos desfechos foram doenças e acidentes não é surpreendente, se considerada a tendência no campo da saúde de se ater à doença ${ }^{28}$. Essa tendência é reforçada na área de saúde do trabalhador, conforme sinalizaram Gomez e Lacaz ${ }^{19}$, porque conceitos e práticas da Medicina do Trabalho e da Saúde Ocupacional orientam grande parte da produção cientifica. Análises focadas no modelo "um risco-uma doença" contrariam as direções das problemáticas afeitas ao marco de referência que leva em conta a teoria social ou o conceito de processo de produção. A literatura internacional incorporou hipóteses relacionadas aos efeitos das atuais dinâmicas do mercado de trabalho. Os resultados indicam que desempregados ou subempregados não são cobertos pela previdência social carecem de recurso para comprar os serviços de saúde e de direitos políticos para ter acesso a esses serviços via cidadania ${ }^{9-13}$.

É positivo observar, ainda que representando uma pequena fração, artigos interessados em conhecer aspectos do absenteísmo e da violência no trabalho. Vale lembrar que a violência é um problema de saúde ocupacional reconhecido, sendo predominante no setor de serviços (saúde, educação ${ }^{29}$. Quanto ao absenteísmo, está reconhecido o seu valor como indicador das condições de saúde e trabalho ${ }^{30}$.

Poucos artigos apresentaram objetivos de estudar aspectos relacionados à vigilância em saúde do trabalhador. Estudar exposição e condições de trabalho seria uma maneira de identificar fatores modificáveis por programas e ações específicas ${ }^{31,32}$. Identificou-se o interesse na abordagem da adesão às práticas de promoção da saúde e na elaboração de problemáticas que expressam referenciais sobre a subjetividade e relações interpessoais no trabalho, ainda que minoritária a proporção de artigos estruturados sob esse eixo. Contudo cabe aqui a necessária inflexão sobre o caráter quantitativo que estrutura os inquéritos de maneira a não autorizar críticas sobre a ausência de abordagens compreensivas, pois essas são próprias aos estudos qualitativos que não fazem parte do corpus de que trata essa revisão.
O crescente interesse dos pesquisadores nas dimensões sociais das desigualdades interessa aos agentes da saúde pública uma vez identificadas diferenças da distribuição dos eventos entre os grupos populacionais e no interior de um mesmo grupo, ao que se convencionou nominar de determinantes sociais da saúde ${ }^{1}$. Contudo, os Inquéritos Ocupacionais de que tratam os artigos dessa revisão reproduzem, em sua maioria, o ponto de vista sobre a saúde, o trabalho e os trabalhadores que foi desenvolvido no século passado: uma categoria profissional empregada (mais ou menos protegida pela estrutura previdenciária e trabalhista) atuando no locus de uma empresa e vulnerável a doenças passíveis de certificação médica por estar exposta aos riscos ambientais específicos. Esse modelo assentado no paradigma fordista embasou não somente a gestão das empresas, mas também a atuação sindical e dos agentes do poder público. Não é surpreendente que em algum nível provoque influências sobre a prática acadêmica e científica ${ }^{19}$.

Os sistemas de informação do Ministério da Saúde (Sistema de Informação de Mortalidade (SIM), Sistema Nacional de Agravos de Notificação (SINAN), do Ministério da Previdência Social (Dataprev) e do Ministério do Trabalho (RAIS) são de fácil acesso ${ }^{33}$. Contudo, há críticas sobre a abrangência, completude e qualidade dos dados disponíveis. Dessa feita, estão justificados os estudos que buscam produzir dados próprios. Mas esses seriam suficientes para explorar as hipóteses cabíveis? Ou seria o caso de convocar uma agenda de pesquisa preocupada em obter meios para planejar e articular os sistemas de informação existentes a fim de produzir informações abrangentes e válidas para embasar decisões? A experiência na saúde pública tem aconselhado fortalecer essa estratégia ${ }^{34}$. $\mathrm{Na}$ área de saúde do trabalhador, seria viável avançar em busca de articulação dos setores saúde, trabalho e previdência? ${ }^{19}$ Iniciativas em curso buscam o rompimento de fronteiras, como se vê na Pesquisa Nacional de Saúde, que introduziu módulos específicos sobre situação de emprego e condições de trabalho ${ }^{21}$.

A presente revisão sistemática buscou identificar a estrutura e a qualidade técnica de artigos sobre Inquéritos Ocupacionais no Brasil. Tal estratégia reproduz a linha de pesquisadores hispanófonos ${ }^{3}$, mas é inédita no Brasil. Ademais, é pioneira ao empregar para fins de avaliação metodológica o tradicional Prisma. Vale lembrar que o modelo Prisma é amplamente utilizado na grande área da saúde para examinar resultados empíricos de pesquisas no campo ${ }^{20}$. Utilizou-se desta vez o 
Prisma para avaliar concepções estruturais e metodológicas das pesquisas publicadas nos artigos, sendo portanto admitidas críticas quanto ao desvio do instrumento para fins adjacentes.

Os descritores utilizados na estratégia de busca foram escolhidos na tentativa de englobar o maior número possível de artigos. É possível, entretanto, falhas nas definições com repercussões sobre o alcance do corpus. As escolhas de busca trouxeram restrições porque o objeto de que trata os Inquéritos Ocupacionais é transversal a outros campos disciplinares, como economia e sociologia do trabalho, cujas bases de indexação de artigos são outras. Dessa feita, são possíveis vieses nas análises sobre o panorama dos Inquéritos Ocupacionais no Brasil. As vantagens da revisão devem ser mencionadas, com destaque para o cuidado na seleção dos artigos e detalhamento tanto dos critérios no processo de construção do corpus, quanto na apresentação e discussão dos resultados.

Os resultados dos Inquéritos Ocupacionais são peças relevantes para maior eficiência na gestão de políticas públicas voltadas à saúde dos trabalhadores. Sugere-se maior atenção quanto ao rigor metodológico, principalmente no tocante ao cálculo amostral e validação de instrumentos.
Por fim, inquietações e dilemas decorrentes das sínteses e interpretações podem ser úteis para fomentar o debate com vistas a fortalecer o campo de estudos em saúde do trabalhador no País.

Os resultados ao indicarem iniquidades na distribuição dos Inquéritos Ocupacionais, tanto setorial quanto regionalmente foram verificadas, sugerem rever o planejamento dos investimentos e incentivos tanto na formação de recursos humanos, quanto em recursos financeiros para apoiar pesquisas ${ }^{35}$. Se os inquéritos são a base da saúde pública, então são necessários esforços para a criação de consensos metodológicos e fomento à pesquisa em setores e regiões menos favorecidas.

Pesquisadores na área e saúde dos trabalhadores estão preocupados diante das decisões do Governo que modificaram a regulamentação das relações de emprego e de trabalho. Diante da nova conjuntura, é maior a responsabilidade em reverter o quadro de fragmentação e dispersão da produção científica na área de saúde do trabalhador, conforme alertado por Gomez e Lacaz ${ }^{19}$ há quase duas décadas. A obra de Thomas Kuhn ${ }^{36}$ esclarece que o progresso científico se efetua não pela acumulação do conhecimento, mas sobretudo pela mudança de ponto de vista.

\section{Colaboradores}

TGM Castro, EP Lima e AA Assunção participaram da concepção e delineamento do artigo. TGM Castro analisou e processou os dados. Todos os autores revisaram o texto final. 


\section{Referências}

1. Hämmig O, Gutzwiller F, Kawachi I. The contribution of lifestyle and work factors to social inequalities in self-rated health among the employed population in Switzerland. Soc Sci Med 2014; 121:74-84.

2. Merino-Salazar P, Artazcoz L, Campos-Serna J, Gimeno D, Benavides FG. National working conditions surveys in Latin America: comparison of methodological characteristics. Int J Environ Health 2015; 21(3):266-274.

3. Artazcoz L. Encuestas sobre condiciones del trabajo: reflexiones y sugerencias. Archivos de Prevención de Riesgos Laborales 2003; 6(1):1-3.

4. Brugulat-Guiteras P, Mompart-Penina A, Séculi-Sánches E, Tresserras-Gaju R, De la Puente-Martorell ML. Encuestas de salud: luces y sombras. Medicina Clínica 2010; 134(Supl. 1):21-26.

5. European Foundation for the Improvement of Living and Working Conditions. European Working Condition Survey. 2012. [acessado 2015 Jul 22]. Disponível em: http://www.eurofound.europa.eu/ewco/surveys/ index.htm

6. Benavides FG, Wesseling C, Delclos GL, Felknor S, Pinilla J, Rodrigo F. Working conditions and health in Central America: a survey of 12024 workers in six countries. Occup Environ Med 2014; 71(7):459-465.

7. Instituto Brasileiro de Geografia e Estatística (IBGE). Pesquisa Nacional por Amostra de Domicílios Contínua Mercado de Trabalho Conjuntural Divulgação Mensal. Rio de Janeiro: IBGE; 2015. [acessado 2016 Ago 2]. Disponível em: Disponível em: ftp://ftp.ibge.gov.br/ Trabalho_e_Rendimento/Pesquisa_ Nacional_por_ Amostra_de_Domicilios_continua/Mensal/Comentarios/pnadc_201508_comentarios.pdf

8. Oliveira T, Proni MW. Mercado de trabalho heterogêneo e flexível: um problema persistente no Brasil. Revista da ABET 2016; 15(2):60-86.

9. Caroli E, Godard M. Does job insecurity deteriorate health? Health Economics 2016; 25(2):131-147.

10. Buffet M-A, Priha E. Workforce diversity and risk assessment - Ensuring everyone is covered. European Agency for Safety and Health at Work, Belgium, 2009. [acessado 2015 Jul 22]. Disponível em: http://osha. europa.eu/en/publications/reports/TE7809894ENC

11. Schenker MB. A global perspective of migration and occupational health. Am J Ind Med 2010; 53(4):329337.

12. Krieger N. Workers are people too: societal aspects of occupational health disparities - an ecosocial perspective. Am J Ind Med 2010; 53(2):104-115.

13. Barnay T. Health, work and working conditions: a review of the European economic literature. Eur $J$ Health Econ 2016; 17(6):693.

14. Lima EP, Assunção AA, Barreto SM. Prevalência de depressão em bombeiros. Cad Saude Publica 2015; 31(4):733-743

15. Weiler A. Working Conditions Survey: a comparative analysis. Dublin: European Foundation for the Improvement of Living and Working Conditions; 2007. [acessado 2015 Ago 15]. Disponível em: http://www. eurofound.europa.eu/publications/htmlfiles/ ef0744. htm
16. Benavides FG, Merino-Salazar P, Cornelio C, Avila A, Agudelo-Suáres A, Amable M, Artazcoz L, Astete J, Barraza D, Berhó F, Milián LC, Delclòs G, Funcasta L, Gerke J, Gimeno D, Itatí-Iñiguez MJ, Lima EP, Martínez-Iñigo D, Medeiros AM, Orta L, Pinilla J, Rodrigo F, Rojas M, Sabastizagal I, Vallebuona C, Vermeylen G, Villalobos GH, Vives A. Cuestionario y criterios metodológicos para Encuestas sobre Condiciones de Trabajo, Empleo y Salud en América Latina y el Caribe. Cad Saude Publica 2016; 32(9):e00210715.

17. Aday LA, Cornelius LJ. Designing and conducting health surveys: a compreensive guide. $3^{\text {rd }}$ ed. San Francisco: Jossey Bass; 2006.

18. Narocki C, Zimmerman M, Artazcoz L, Gimeno D, Benavides FG. Encuestas de condiciones de trabajo y salud en España: comparación de los contenidos del cuestionario del trabajador. Archivos de Prevención de Riesgos Laborales 2009; 12:14-18.

19. Gomez CM, Lacaz FAC. Saúde do trabalhador: novas-velhas questões. Cien Saude Colet 2005; 10(4):797807.

20. Moher D, Liberati A, Tetzlaff J, Altman DG, The PRISMA Group. Preferred Reporting items for systematic reviews and meta-analyses: the PRISMA Statement. Ann Intern Med 2009; 151(4):264-269, W64.

21. Malta DC, Leal MC, Costa MFL, Morais Neto OL. Inquéritos Nacionais de Saúde: experiência acumulada e proposta para o inquérito de saúde brasileiro. Revista Brasileira de Epidemiologia 2008; 11(Supl. 1):159-167.

22. Brasil. Ministério da Ciência, Tecnologia e Inovação (MCTI). Coordenação-Geral de Indicadores (CGIN) - ASCAV/SEXEC. Sistema Integrado de Administração Financeira do Governo Federal (Siafi). Extração especial realizada pelo Serviço Federal de Processamento de Dados (Serpro) e Balanços Gerais dos Estados. Dispêndios públicos em pesquisa e desenvolvimento $(P \ll D)$, por objetivo socioeconômico, 20002013. Brasília: MCTI; 2015. [acessado 2016 Ago 2]. Disponível em: http://www.mct.gov.br/index.php/ content/view/9134/Brasil_Dispendios_publicos_em_ pesquisa_e_desenvolvimento_P_D_por_objetivo_socioeconomico.html.

23. Guimarães R. Pesquisa em saúde no Brasil. Rev Saude Publica 2006; 40(Esp.):3-10.

24. Todos pela educação; Editora Moderna. Anuário Brasileiro da Educação Básica 2016. São Paulo: Moderna, 2016. [acessado 2016 Jul 25]. Disponível em: http:// www.todospelaeducacao.org.br//arquivos/ biblioteca/ anuario_educacao_2016.pdf.

25. Centro de Gestão de Estudos Estratégicos (CGEE). Mestres e doutores 2015. Estudos da demografia da base técnico científica brasileira. Brasília, 2016. [acessado 2016 Jul 28]. Disponível em: https://www.cgee. org.br/documents/10195/734063/ Apres_CGEE_ MD2015_SBPCvfrev.pdf/d50b9e9d-5f0f-4b40-af53562 cf8fa605a.

26. Campos-Serna, J, Molina AA, Pinilla J, Benavides FG. Recomendaciones metodológicas para el diseño de encuestas de condiciones de trabajo y salud. Archivos de Prevención de Riesgos Laborales 2009; 12(4):195198. 
27. Iñiguez MJI. Encuestas de condiciones de trabajo y salud: su utilizacíon em la investigacíon em salud laboral. Medicina y Seguridad del Trabajo 2012; 58(228):205-215.

28. Almeida Filho N. A ciência da saúde. São Paulo: Hucitec; 2000.

29. Piquero NL, Piquero AR, Craig JM, Clipper SJ. Assessing research on workplace violence 2000-2012. Aggression and Violent Behavior 2013; 18(3):383-394.

30. Griep RH, Rotenberg L, Chor D, Landsbergis P. Beyond simple approaches to studying the association between work characteristics and absenteeism: Combining DCS and the ERI models. Work and Stress 2010: 24:179-195.

31. Lacaz FAC. O campo saúde do trabalhador: resgatando conhecimentos e práticas sobre as relações trabalho-saúde. Cad Saude Publica 2007; 23(4):757-766.

32. Machado JMH. A propósito da vigilância em saúde do trabalhador. Cien Saude Colet 2005; 10(4):987-992.

33. Facchini LA, Nobre LCC, Faria NMX, Fassa AG, Thumé E, Tomasi E, Santana V. Sistema de Informação em Saúde do Trabalhador: desafios e perspectivas para o SUS. Cien Saude Colet 2005; 10(4):857-867.

34. Santana VS, Nobre LCC, Waldvogel BC. Acidentes de trabalho no Brasil entre 1994 e 2004: uma revisão. Cien Saude Colet 2005; 10(4):841-885.

35. Almeida ECE, Guimarães JA. Brazil's growing production of scientific articles: how are we doing with review articles and other qualitative indicators? Scientometrics 2013; 97(2):287-315.

36. Kuhn T. A estrutura das revoluções científicas. São Paulo: Perspectiva; 2006.

Artigo apresentado em 11/11/2016

Aprovado em 11/02/2018

Versão final apresentada em 13/02/2018 